\title{
Interactive comment on "Air quality and health benefits from ultra-low emission control policy indicated by continuous emission monitoring: $A$ case study in the Yangtze River Delta region, China" by Yan Zhang et al.
}

Anonymous Referee \#2

Received and published: 10 December 2020

Under the big pressure of air quality improvement, China has been conducting a series of measures to reduce the emissions of air pollutants, including the "ultra-low" emission policy for power and specific industrial sectors. Continuous emission monitoring system (CEMS) has been gradually installed and operated to examine the real emission status of individual plants. Besides, CEMS provided opportunities of improving the understanding of air pollutant emissions for atmospheric science community. Focusing on the YRD, one of the most economically developed region in China, this paper presented an extended study based on the previously developed emission inventory of 
power sector using the CEMS measurement (Y. Zhang et al., 2019). The authors applied air quality model and evaluated the YRD emission inventory with CEMS incorporated. They further combined the air quality model and exposure-response model, and analyzed the benefit of ultra-low emission policy on air quality and health. It provides the evidence of emission data improvement and the environmental health implication of current air pollution control policy, thus it fits the scope of Atmos Chem Phys. In general the paper is well organized and clearly written. I would suggest its acceptance for publication, with minor revisions or discussions conducted on the following issues.

1. Abstract. Some sentences are unclear and should be rewritten. Lines 49-50: "11\%, $7 \%$ and $2 \%$ of SO2, NOX and PM" for which case?

2. Lines 155-157, did Gao et al. (2018) (and some other studies maybe) include the CEMS data for emission inventory development and stress the ultra-low emission policy?

3. Lines 226-234. It seems that you apply the inventory by Xia et al. (2016) but the spatiotemporal distribution of MEIC? Why not use MEIC directly?

4. Lines 251-253. Some information is missing here. Case 4 assumes both power and industrial boilers would meet the ultra-low emission limit. Do the two sectors share the same limit? In your previous work (Y. Zhang et al., 2019) you analyzed the emission limit for power sector with CEMS incorporated, but how about references for industrial boilers? Description should be given here.

5. Table 1 seems unnecessary, while the emission data in difference cases are more important (Table S2). Could you combine Table 1 and Table S2?

6. Line 326-328. The difference for SO2 was $10 \%$, did this contradict the statement in lines 45-46 in the abstract: "... SO2, NO2, O3 and PM2.5 concentrations compared to those of Case 2, our base case for policy comparisons, were less than $7 \%$ for all pollutants"?

Printer-friendly version

Discussion paper 
7. Lines 359-360. I cannot quite understand the numbers. You are comparing NMBs and NMEs for two cases, then what did the two groups of numbers exactly stand for?

8. Line 367. Is it commonly known that the YRD is under VOC-limited regime in terms of $\mathrm{O} 3$ formation? At least some literatures should be provided here.

9. Lines 435-437. Did the "ultra-low" emission policy include the limit of VOCs? Or did CEMS include the information of VOCs? Some explanations should be given here.

comment

10. Lines 539-540: Any reason could be provided?

11. Language issues.

Line 119: "environmental concentrations"? Line 125: delete the word "itself"; Line 504: change "sectors" to "boilers"; Lines 531-534: rewrite the sentence

Interactive comment on Atmos. Chem. Phys. Discuss., https://doi.org/10.5194/acp-2020-818, 2020. 